Claremont Colleges

Scholarship@ Claremont

All HMC Faculty Publications and Research

HMC Faculty Scholarship

4-1-2002

\title{
A Stirling Encounter with Harmonic Numbers
}

Arthur T. Benjamin

Harvey Mudd College

Gregory O. Preston '01

Harvey Mudd College

Jennifer J. Quinn

Occidental College

\section{Recommended Citation}

Benjamin, Arthur T., Greg O. Preston and Jennifer J. Quinn. "A Stirling Encounter with Harmonic Numbers." Mathematics Magazine, Vol. 75, No. 2, pp. 95-103, April 2002.

This Article is brought to you for free and open access by the HMC Faculty Scholarship at Scholarship @ Claremont. It has been accepted for inclusion in All HMC Faculty Publications and Research by an authorized administrator of Scholarship @ Claremont. For more information, please contact scholarship@cuc.claremont.edu. 


\section{D.MA}

A Stirling Encounter with Harmonic Numbers

Author(s): Arthur T. Benjamin, Gregory O. Preston and Jennifer J. Quinn

Source: Mathematics Magazine, Vol. 75, No. 2 (Apr., 2002), pp. 95-103

Published by: Mathematical Association of America

Stable URL: http://www.jstor.org/stable/3219141

Accessed: 11/06/2013 17:34

Your use of the JSTOR archive indicates your acceptance of the Terms \& Conditions of Use, available at

http://www.jstor.org/page/info/about/policies/terms.jsp

JSTOR is a not-for-profit service that helps scholars, researchers, and students discover, use, and build upon a wide range of content in a trusted digital archive. We use information technology and tools to increase productivity and facilitate new forms of scholarship. For more information about JSTOR, please contact support@jstor.org.

Mathematical Association of America is collaborating with JSTOR to digitize, preserve and extend access to Mathematics Magazine. 


\title{
A Stirling Encounter with Harmonic Numbers
}

\author{
ARTHUR T. BENJAMIN \\ GREGORY O. PRESTON \\ Harvey Mudd College \\ Claremont, CA 91711 \\ benjamin@hmc.edu \\ gpreston@hmc.edu \\ JENNIFER J. QUINN \\ Occidental College \\ 1600 Campus Road \\ Los Angeles, CA 90041 \\ jquinn@oxy.edu
}

Harmonic numbers are defined to be partial sums of the harmonic series. For $n \geq 1$, let

$$
H_{n}=1+\frac{1}{2}+\frac{1}{3}+\cdots+\frac{1}{n} .
$$

The first five harmonic numbers are $H_{1}=1, H_{2}=3 / 2, H_{3}=11 / 6, H_{4}=25 / 12$, $H_{5}=137 / 60$. For convenience we define $H_{0}=0$. Since the harmonic series diverges, $H_{n}$ can get arbitrarily large, although it does so quite slowly. For instance, $H_{1,000,000} \approx$ 14.39 .

Harmonic numbers even appear in real life. If you stack 2-inch long playing cards to overhang the edge of a table as far as possible, the maximum distance that $n$ cards can hang off the edge of the table is $H_{n}$ [5]. For example, 4 cards can be stacked to extend past the table by just over 2 inches, since $H_{4}=25 / 12$.

Harmonic numbers satisfy many interesting properties. For nonnegative integers $n$ and $m$, we list some identities below:

$$
\begin{aligned}
\sum_{k=1}^{n-1} H_{k} & =n H_{n}-n . \\
\sum_{k=m}^{n-1}\left(\begin{array}{c}
k \\
m
\end{array}\right) H_{k} & =\left(\begin{array}{c}
n \\
m+1
\end{array}\right)\left(H_{n}-\frac{1}{m+1}\right) . \\
\sum_{k=m}^{n-1}\left(\begin{array}{l}
k \\
m
\end{array}\right) \frac{1}{n-k} & =\left(\begin{array}{c}
n \\
m
\end{array}\right)\left(H_{n}-H_{m}\right) .
\end{aligned}
$$

Although all of these identities can be proved by algebraic methods (see [5]), the presence of binomial coefficients suggests that these identities can also be proved combinatorially. A combinatorial proof is a counting question, which when answered two different ways, yields both sides of the identity. Combinatorial proofs often provide intuitive and concrete explanations where algebraic proofs may not. For example

$$
\sum_{k=1}^{n-1} k \cdot k !=n !-1
$$

is a standard exercise in mathematical induction. But to a combinatorialist this identity counts permutations in two different ways. The right side counts the number of ways 
to arrange the numbers 1 through $n$, excluding the natural arrangement $123 \ldots n$. The left side counts the same quantity by conditioning on the first number that is not in its natural position: for $1 \leq k \leq n-1$, how many arrangements have $n-k$ as the first number to differ from its natural position? Such an arrangement begins as $123 \ldots n-$ $k-1$ followed by one of $k$ numbers from the set $\{n-k+1, n-k+2, \ldots, n\}$. The remaining $k$ numbers (now including the number $n-k$ ) can be arranged $k$ ! ways. Thus there are $k \cdot k$ ! ways for $n-k$ to be the first misplaced number. Summing over all feasible values of $k$ yields the left side of the identity.

Although $H_{n}$ is never an integer for $n>1$ [5], it can be expressed as a rational number whose numerator and denominator have combinatorial significance. Specifically, for $n \geq 0$ we can always write

$$
H_{n}=\frac{p_{n}}{n !}
$$

as a (typically nonreduced) fraction where $p_{n}$ is a nonnegative integer.

Now $p_{0}=H_{0}=0$. For $n \geq 1, H_{n}=H_{n-1}+1 / n$ leads to

$$
\frac{p_{n}}{n !}=\frac{p_{n-1}}{(n-1) !}+\frac{1}{n}=\frac{n p_{n-1}+(n-1) !}{n !} \text {. }
$$

Hence for $n \geq 1$,

$$
p_{n}=n p_{n-1}+(n-1) !
$$

The combinatorial interpretation of these numbers is the topic of the next section.

\section{Stirling numbers}

For integers $n \geq k \geq 1$, let $\left[\begin{array}{l}n \\ k\end{array}\right]$ denote the number of permutations of $n$ elements with exactly $k$ cycles. Equivalently $\left[\begin{array}{l}n \\ k\end{array}\right]$ counts the number of ways for $n$ distinct people to sit around $k$ identical circular tables, where no tables are allowed to be empty. $\left[\begin{array}{l}n \\ k\end{array}\right]$ is called the (unsigned) Stirling number of the first kind. As an example, $\left[\begin{array}{l}3 \\ 2\end{array}\right]=3$ since one person must sit alone at a table and the other two have one way to sit at the other table. We denote these permutations by (1)(23), (13)(2), and (12)(3). $n \geq 1$,

We can compute the numbers $\left[\begin{array}{l}n \\ k\end{array}\right]$ recursively. From their definition, we see that for

$$
\left[\begin{array}{l}
n \\
1
\end{array}\right]=(n-1) !
$$

since the arrangement $\left(a_{1} a_{2} a_{3} \ldots a_{n}\right)$ is the same as arrangements $\left(a_{2} a_{3} \ldots a_{n} a_{1}\right)$ and $\left(a_{3} a_{4} \ldots a_{1} a_{2}\right)$ and so on. Now for $k \geq 2$, we will see that

$$
\left[\begin{array}{c}
n+1 \\
k
\end{array}\right]=\left[\begin{array}{c}
n \\
k-1
\end{array}\right]+n\left[\begin{array}{l}
n \\
k
\end{array}\right] .
$$

On the left, we are directly counting the number of ways to seat $n+1$ people around $k$ circular tables. On the right we count the same thing while conditioning on what happens to person $n+1$. If $n+1$ is to be alone at a table, then the remaining $n$ people can be arranged around $k-1$ tables in $\left[\begin{array}{c}n \\ k-1\end{array}\right]$ ways. If $n+1$ is not to be alone, then we first arrange 1 through $n$ around $k$ tables (there are $\left[\begin{array}{l}n \\ k\end{array}\right]$ ways to do this); for each of these configurations, we insert person $n+1$ to the right of any of the $n$ already-seated 
people. This gives us $n\left[\begin{array}{l}n \\ k\end{array}\right]$ different permutations where $n+1$ is not alone. Summing gives equation (6).

Notice that when $k=2$, equation (6) becomes

$$
\left[\begin{array}{c}
n+1 \\
2
\end{array}\right]=n\left[\begin{array}{l}
n \\
2
\end{array}\right]+(n-1) !,
$$

which is the same as recurrence (5) with $p_{n}=\left[\begin{array}{c}n+1 \\ 2\end{array}\right]$. Since $p_{1}=1=\left[\begin{array}{l}2 \\ 2\end{array}\right]$, it follows that for all $n \geq 1, p_{n}=\left[\begin{array}{c}n+1 \\ 2\end{array}\right]$. Combining with the definition of $p_{n}$ in (4) gives

THEOREM 1. For $n \geq 1$,

$$
H_{n}=\frac{1}{n !}\left[\begin{array}{c}
n+1 \\
2
\end{array}\right] .
$$

Next we show how to count Theorem 1 directly-without relying on a recurrence. First we set some notational conventions. Let $\mathcal{T}_{n}$ denote the set of arrangements of the numbers 1 through $n$ into two disjoint, nonempty cycles. Thus $\left|\mathcal{T}_{n}\right|=\left[\begin{array}{c}n \\ 2\end{array}\right]$. We always write our cycles with the smallest element first, and list the cycles in increasing order according to the first element. For example, $\mathcal{T}_{9}$ includes the permutation (185274)(396), but not (195)(2487)(36) nor (123)(4567)(8)(9). By our convention, the cycle containing 1 is always written first; consequently we call it the left cycle. The remaining cycle is called the right cycle. All permutations in $\mathcal{T}_{n}$ are of the form $\left(a_{1} a_{2} \ldots a_{j}\right)\left(a_{j+1} \ldots a_{n}\right)$, where $1 \leq j \leq n-1, a_{1}=1$, and $a_{j+1}$ is the smallest element of the right cycle.

For a purely combinatorial proof of Theorem 1 that does not rely on a recursion, we ask, for $1 \leq k \leq n$, how many permutations of $\mathcal{T}_{n+1}$ have exactly $k$ elements in the right cycle? To create such a permutation, first choose $k$ elements from $\{2, \ldots, n+1\}$ $\left(\left(\begin{array}{l}n \\ k\end{array}\right)\right.$ ways), arrange these elements in the right cycle $((k-1)$ ! ways), then arrange the remaining $n-k$ elements in the left cycle following the number $1((n-k)$ ! ways). Hence there are $\left(\begin{array}{l}n \\ k\end{array}\right)(k-1) !(n-k) !=n ! / k$ permutations of $\mathcal{T}_{n+1}$ with $k$ elements in the right cycle. Since $\mathcal{T}_{n+1}$ has $\left[\begin{array}{c}n+1 \\ 2\end{array}\right]$ permutations, it follows that

$$
\left[\begin{array}{c}
n+1 \\
2
\end{array}\right]=\sum_{k=1}^{n} \frac{n !}{k}=n ! H_{n},
$$

as desired.

Another way to prove Theorem 1 is to show that for $2 \leq r \leq n+1$, there are $\frac{n !}{r-1}$ permutations in $\mathcal{T}_{n+1}$ that have $r$ as the minimum element of the right cycle.

Here, the permutations being counted have the form $(1 \ldots)(r \ldots)$ where elements 1 through $r-1$ all appear in the left cycle, and elements $r+1$ through $n+1$ can go in either cycle. To count this, arrange elements 1 through $r-1$ into the left cycle, listing element 1 first; there are $(r-2)$ ! ways to do this. Place element $r$ into the right cycle. Now we insert elements $r+1$ through $n+1$, one at a time, each immediately to the right of an already placed element. In this way, elements 1 and $r$ remain first (and smallest) in their cycles. Specifically, the element $r+1$ can go to the right of any of the elements 1 through $r$. Next, $r+2$ can go to the right of any of the elements 1 through $r+1$. Continuing in this way, the number of ways to insert elements $r+1$ through $n+1$ is $r(r+1)(r+2) \cdots n=n ! /(r-1)$ !. This process creates a permutation in $\mathcal{T}_{n+1}$ with $r$ as the smallest element in the right cycle. Thus, there are

$$
(r-2) ! \frac{n !}{(r-1) !}=\frac{n !}{r-1}
$$


such permutations. Since $\mathcal{T}_{n+1}$ has $\left[\begin{array}{c}n+1 \\ 2\end{array}\right]$ permutations, and every permutation in $\mathcal{T}_{n+1}$ must have some smallest integer $r$ in the right cycle, where $2 \leq r \leq n+1$, we get

$$
\left[\begin{array}{c}
n+1 \\
2
\end{array}\right]=\sum_{r=2}^{n+1} \frac{n !}{r-1}=n ! \sum_{k=1}^{n} \frac{1}{k}=n ! H_{n} .
$$

An alternate way to see that $n ! /(r-1)$ counts permutations of the form $(1 \cdots)(r \cdots)$ is to list the numbers 1 through $n+1$ in any order with the provision that 1 be listed first. There are $n$ ! ways to do this. We then convert our list $1 a_{2} a_{3} \cdots r \cdots a_{n+1}$ to the permutation $\left(1 a_{2} a_{3} \cdots\right)\left(r \cdots a_{n+1}\right)$ by inserting parentheses. This permutation satisfies our conditions if and only if the number $r$ is listed to the right of elements $2,3, \ldots, r-1$. This has probability $1 /(r-1)$ since any of the elements $2,3, \ldots, r$ have the same chance of being listed last among them. Hence the number of permutations that satisfy our conditions is $n ! /(r-1)$.

Algebraic connection The Stirling numbers can also be defined as coefficients in the expansion of the rising factorial function [3]:

$$
x(x+1)(x+2) \cdots(x+n-1)=\sum_{m=1}^{n}\left[\begin{array}{l}
n \\
m
\end{array}\right] x^{m} .
$$

Using this definition, Theorem 1 can be derived algebraically by computing the $x^{2}$ coefficient of $x(x+1)(x+2) \cdots(x+n)$.

To show that this algebraic definition of Stirling numbers is equivalent to the combinatorial definition, one typically proves that both satisfy the same initial conditions and recurrence relation. However, a more direct correspondence exists [1], which we illustrate with an example.

By the algebraic definition, the Stirling number $\left[\begin{array}{c}10 \\ 3\end{array}\right]$ is the coefficient of $x^{3}$ in the expansion $x(x+1)(x+2) \cdots(x+9)$. The combinatorial definition says $\left[\begin{array}{c}10 \\ 3\end{array}\right]$ counts the number of ways that elements $0,1,2, \ldots, 9$ can sit around 3 identical circular tables. Why are these definitions the same? Each term of the $x^{3}$ coefficient is a product of seven numbers chosen from among 1 through 9 . Surely this must be counting something. What is a term like $1 \cdot 2 \cdot 3 \cdot 5 \cdot 6 \cdot 8 \cdot 9$ counting?

As illustrated in FIGURE 1, this counts the number of ways elements 0 through 9 can seat themselves around 3 identical tables where the smallest elements of the tables are the "missing" numbers 0,4 , and 7 . To see this, we pre-seat numbers $0,4,7$ then seat the remaining numbers one at a time in increasing order. The number 1 has just one option-sit next to 0 . The number 2 then has two options-sit to the right of 0 or sit to the right of 1 . The number 3 now has three options-sit to the right of 0 or 1 or 2 . The number 4 is already seated. Now number 5 has five options-sit to the right of 0 or 1 or 2 or 3 or 4 , and so on. A general combinatorial proof of equation (8) can also be done by the preceding (or should that be "pre-seating"?) argument.

With this understanding of the interactions between harmonic and Stirling numbers, we now provide combinatorial explanations of other harmonic identities.

\section{Recounting harmonic identities}

In this section, we convert identities (1), (2), and (3) into statements about Stirling numbers and explain them combinatorially. We view each identity as a story of a counting problem waiting to be told. Each side of the identity recounts the story in a different, but accurate way. Both of our combinatorial proofs of Theorem 1 were 


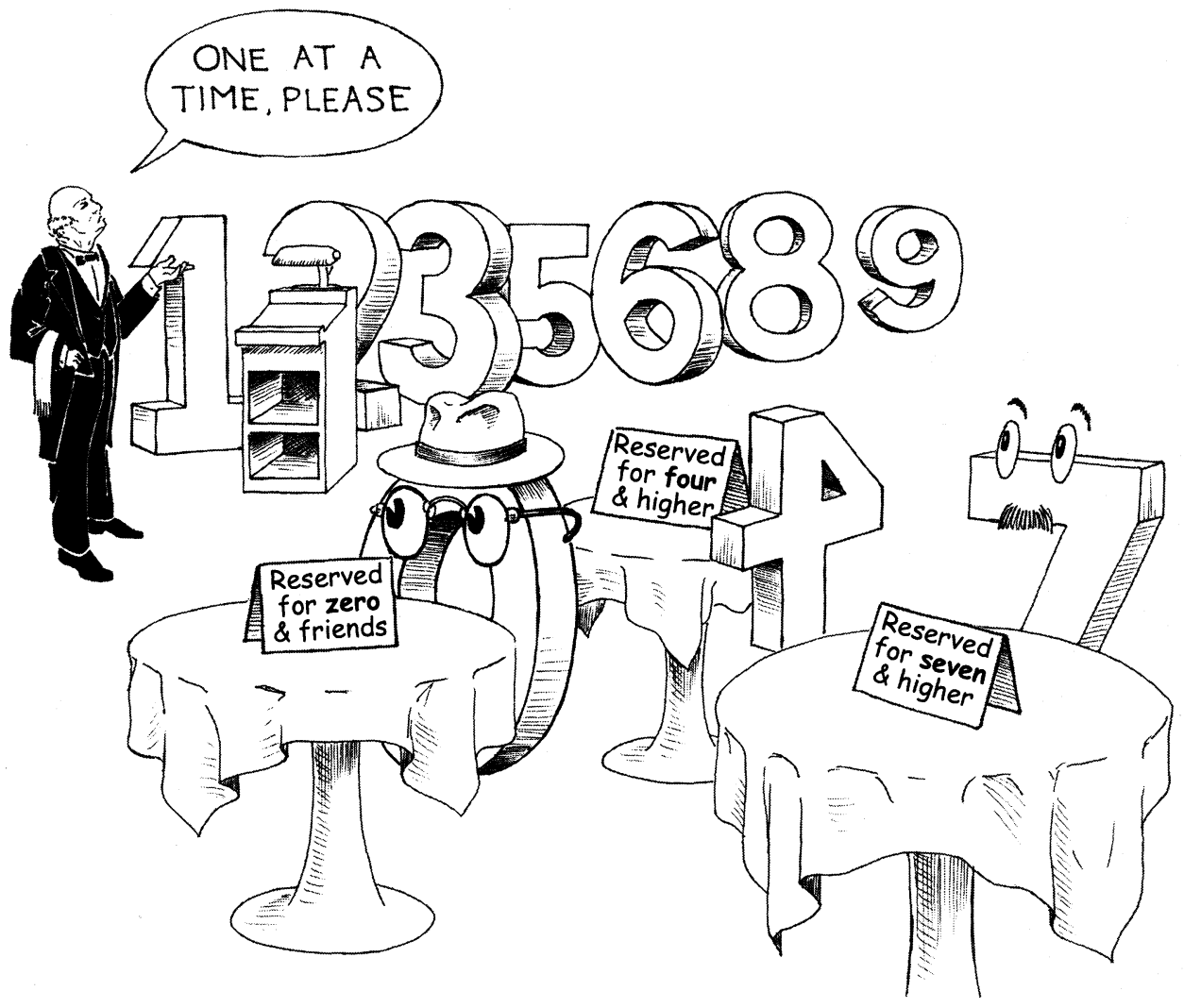

Figure 1 How many ways can the numbers 1, 2, 3, 5, 6, 8, 9 seat themselves around these tables?

obtained by partitioning the set $\mathcal{T}_{n+1}$ according to the size of the right cycle or the minimum element of the right cycle, respectively. In what follows, we shall transform harmonic equations (1), (2) and (3) into three Stirling number identities, each with $\left[\begin{array}{l}n \\ 2\end{array}\right]$ on the left-hand side. The right-hand sides will be combinatorially explained by partitioning $\mathcal{T}_{n}$ according to the location of element 2 , the largest of the last $t$ elements, or the neighborhood of the elements 1 through $m$. Our first identity, after applying Theorem 1 , and re-indexing $(n:=n-1)$ gives us

IDENTITY 1. For $n \geq 2$

$$
\left[\begin{array}{l}
n \\
2
\end{array}\right]=(n-1) !+\sum_{k=1}^{n-2} \frac{(n-2) !}{k !}\left[\begin{array}{c}
k+1 \\
2
\end{array}\right] .
$$

To prove this combinatorially, we note that the left side of the identity, $\left[\begin{array}{l}n \\ 2\end{array}\right]$, counts the number of permutations in $\mathcal{T}_{n}$. On the right, we know from our second combinatorial proof of Theorem 1, that $(n-1)$ ! counts the number of permutations in $\mathcal{T}_{n}$ where the number 2 appears in the right cycle. It remains to show that the summation above counts the number of permutations in $\mathcal{T}_{n}$ where 2 is in the left cycle. Any such permutation has the form

$$
\left(1 a_{1} a_{2} \cdots a_{n-2-k} 2 b_{1} b_{2} \cdots b_{j-1}\right)\left(b_{j} \cdots b_{k}\right),
$$

for some $1 \leq k \leq n-2$ and $1 \leq j \leq k$. We assert that the number of these permutations with exactly $k$ terms to the right of 2 is given by the $k$ th term of the sum. 
To see this, select $a_{1}, a_{2}, \ldots, a_{n-2-k}$ from the set $\{3, \ldots, n\}$ in any of $(n-2) ! / k$ ! ways. From the unchosen elements, there are $\left[\begin{array}{c}k+1 \\ 2\end{array}\right]$ ways to create two nonempty cycles of the form $\left(2 b_{1} \ldots b_{j-1}\right)\left(b_{j} \ldots b_{k}\right)$ where $1 \leq j \leq k$. Multiplying the two counts gives the $k$ th term of the sum as the number of permutations in $\mathcal{T}_{n}$ with exactly $k$ terms to the right of 2 , as was to be shown.

We apply a different combinatorial strategy to prove the more general equation (2), which, after applying Theorem 1 and re-indexing $(n:=n-1, m:=t-1$, and $k:=k-2$ ), gives us

$$
\begin{aligned}
& \text { IDENTITY 2. For } 1 \leq t \leq n-1 \\
& \left.\qquad \begin{array}{l}
n \\
2
\end{array}\right]=\frac{(n-1) !}{t}+t \sum_{k=t+1}^{n}\left[\begin{array}{c}
k-1 \\
2
\end{array}\right] \frac{(n-1-t) !}{(k-1-t) !}
\end{aligned}
$$

The combinatorial proof of this identity requires a new interpretation of $(n-1) ! / t$. For $1 \leq t \leq n-1$, we define the last $t$ elements of $\left(1 a_{2} \cdots a_{j}\right)\left(a_{j+1} \cdots a_{n}\right)$ to be the elements $a_{n}, a_{n-1}, \ldots a_{n+1-t}$, even if some of them are in the left cycle. For example, the last 5 elements of (185274)(396) are 6, 9, 3, 4, and 7.

We claim that for $1 \leq t \leq n-1$, the number of permutations in $\mathcal{T}_{n}$ where the largest of the last $t$ elements is alone in the right cycle is $(n-1) ! / t$. Here, we are counting permutations of the form $\left(1 a_{2} \ldots a_{n-1}\right)\left(a_{n}\right)$, where $a_{n}$ is the largest of $\left\{a_{n+1-t}, a_{n+2-t}, \ldots, a_{n-1}, a_{n}\right\}$. Among all $(n-1)$ ! permutations of this form, the largest of the last $t$ elements is equally likely to be anywhere among the last $t$ positions. Hence $(n-1) ! / t$ of them have the largest of the last $t$ elements in the last position.

Next we claim that for $1 \leq t \leq n-1$, the number of permutations in $\mathcal{T}_{n}$ where the largest of the last $t$ elements is not alone in the right cycle is the summation in Identity 2.

To see this, we count the number of such permutations where the largest of the last $t$ elements is equal to $k$. Since the number 1 is not listed among the last $t$ elements, we have $t+1 \leq k \leq n$. To construct such a permutation, we begin by arranging numbers 1 through $k-1$ into two cycles. Then insert the number $k$ to the right of any of the last $t$ elements. There are $\left[\begin{array}{c}k-1 \\ 2\end{array}\right] t$ ways to do this. The right cycle contains at least one element less than $k$, so $k$ is not alone in the right cycle (and could even be in the left cycle). So that $k$ remains the largest among the last $t$ elements, we insert elements $k+1$ through $n$, one at a time, to the right of any but the last $t$ elements. There are $(k-$ $t)(k+1-t) \cdots(n-1-t)=(n-1-t) ! /(k-1-t)$ ! ways to do this. Multiplying the two counts give the $k$ th term of the sum as the number of permutations where the largest of the last $t$ elements equals $k$, and it is not alone in the right cycle; summing over all possible values of $k$, we count all such permutations. Since for any permutation in $\mathcal{T}_{n}$, the largest of the last $t$ elements is either alone in the last cycle, or it isn't, and this establishes Identity 2.

Notice that when $t=1$, Identity 2 simplifies to Identity 1 . When $t=n-1$, Identity 2 essentially simplifies to equation (7).

For our final identity, we convert equation (3) to Stirling numbers using Theorem 1 and re-indexing $(n:=n-1, m:=m-1$, and $k:=t-1)$. This gives us

IDENTITY 3. For $1 \leq m \leq n$

$$
\left[\begin{array}{l}
n \\
2
\end{array}\right]=\left[\begin{array}{l}
m \\
2
\end{array}\right] \frac{(n-1) !}{(m-1) !}+\sum_{t=m}^{n-1}\left(\begin{array}{c}
t-1 \\
m-1
\end{array}\right) \frac{(m-1) !(n-m) !}{(n-t)} .
$$

To prove this identity combinatorially, we condition on whether numbers 1 through $m$ all appear in the left cycle. First we claim that for $1 \leq m \leq n$, the first term on 
the right in the identity counts the number of permutations in $\mathcal{T}_{n}$ that do not have elements $1,2, \ldots m$ all in the left cycle: For these permutations, the elements 1 through $m$ can be arranged into two cycles in $\left[\begin{array}{c}m \\ 2\end{array}\right]$ ways. Insert the remaining elements $m+1$ through $n$, one at a time, to the right of any existing element, finding that there are $m(m+1) \cdots(n-1)=(n-1) ! /(m-1)$ ! ways to insert these elements. Multiplying the two counts gives the first term of the right-hand side.

To complete the proof, we must show that the summation on the right counts the number of permutations in $\mathcal{T}_{n}$ where elements 1 through $m$ are all in the left cycle. To see this, we claim that for $m \leq t \leq n-1$, the summand counts the permutations described above with exactly $t$ elements in the left cycle and $n-t$ elements in the right cycle. To create such a permutation, we first place the number 1 at the front of the left cycle. Now choose $m-1$ of the remaining $t-1$ spots in the left cycle to be assigned the elements $\{2, \ldots, m\}$. There are $\left(\begin{array}{c}t-1 \\ m-1\end{array}\right)$ ways to select these $m-1$ spots and $(m-1)$ ! ways to arrange elements $2, \ldots, m-1$ in those spots. For example, to guarantee that elements 1, 2, 3, 4 appear in the left cycle of FIGURE 2, we select three of the five open spots in which to arrange $2,3,4$. The insertion of 5, 6, 7, 8, 9 remains. Now there are $(n-m)$ ! ways to arrange elements $m+1$ through $n$ in the remaining spots, but only one out of $n-t$ of them will put the smallest element of the right cycle at the front of the right cycle. Hence, elements $m+1$ through $n$ can be arranged in $(n-m) ! /(n-t)$ legal ways. Multiplying gives the number of ways to satisfy our conditions for a given $t$, and the total is given by the desired summation.

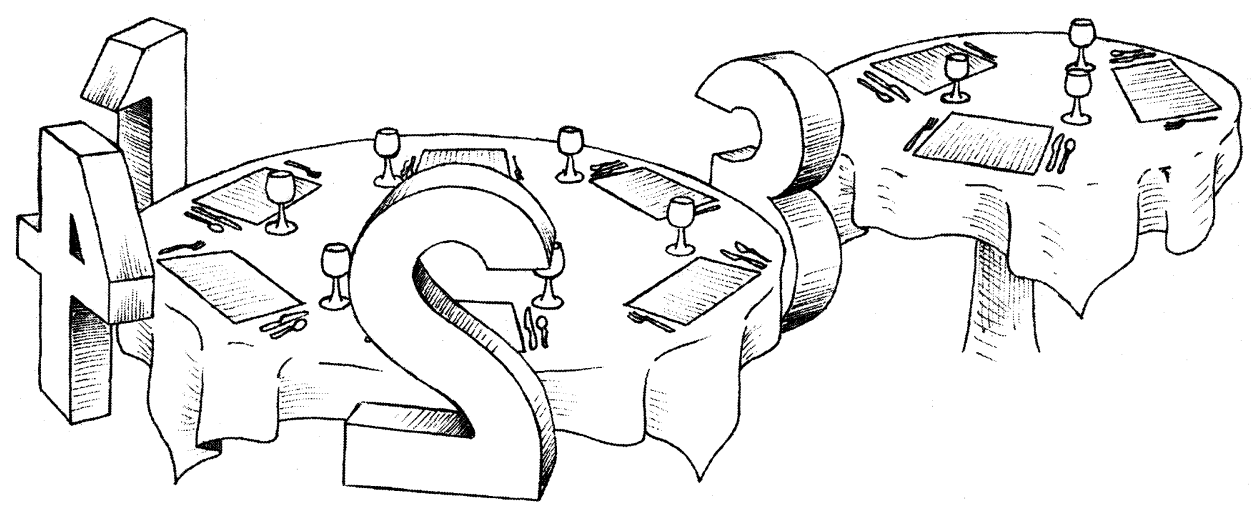

Figure 2 In $\mathcal{T}_{9}$, a permutation with 1,2,3, 4 in a left cycle containing exactly six elements is created by first selecting three of the five open spots, and then arranging 2, 3, 4 in them. Subsequently, 5, 6, 7, 8, 9 will be arranged in the remaining spots.

We have already noted that harmonic numbers arise in real life. A further occurrence arises in calculating the average number of cycles in a permutation of $n$ elements. Specifically,

THEOREM 2. On average, a permutation of n elements has $H_{n}$ cycles.

There are $n$ ! permutations of $n$ elements, of which $\left[\begin{array}{l}n \\ k\end{array}\right]$ have $k$ cycles. Consequently, Theorem 2 says

$$
\frac{\sum_{k=1}^{n} k\left[\begin{array}{l}
n \\
k
\end{array}\right]}{n !}=H_{n},
$$

or equivalently, by Theorem 1 , 
IDENTITY 4. For $n \geq 1$,

$$
\sum_{k=1}^{n} k\left[\begin{array}{l}
n \\
k
\end{array}\right]=\left[\begin{array}{c}
n+1 \\
2
\end{array}\right]
$$

The left side counts the number of permutations of $\{1, \ldots, n\}$ with an arbitrary number of cycles, where one of the cycles is distinguished in some way. For example (1284)(365)(79), (1284)(365)(79), and (1284)(365)(79) are three different arrangements with $k=3$. The right side counts the number of permutations of $\{0,1, \ldots, n\}$ with exactly two cycles. It remains to describe a one-to-one correspondence between these two sets of objects. Can you deduce the correspondence between the following three examples?

$$
\begin{aligned}
& \underline{(1284)}(365)(79) \Longleftrightarrow(079365)(1284) \\
& (1284)(365)(79) \Longleftrightarrow(0791284)(365) \\
& \text { (1284)(365)(79) } \Longleftrightarrow(03651284)(79)
\end{aligned}
$$

In general, we transform the permutation with $n$ elements

$$
\left(C_{k}\right)\left(C_{k-1}\right) \cdots\left(C_{j+1}\right) \underline{\left(C_{j}\right)}\left(C_{j-1}\right) \cdots\left(C_{2}\right)\left(C_{1}\right)
$$

into

$$
\left(0 C_{1} C_{2} \cdots C_{j-1} C_{j+1} \cdots C_{k-1} C_{k}\right)\left(C_{j}\right) .
$$

The process is easily reversed. Given $\left(0 a_{1} \cdots a_{n-j}\right)\left(b_{1} \cdots b_{j}\right)$ in $\mathcal{T}_{n+1}$, the right cycle becomes the distinguished cycle $\left(b_{1} \cdots b_{j}\right)$. The distinguished cycle is then inserted among the cycles $C_{k-1}, \ldots C_{2}, C_{1}$, which are generated one at a time as follows: $C_{1}$ (the rightmost cycle) begins with $a_{1}$ followed by $a_{2}$ and so on until we encounter a number $a_{i}$ that is less than $a_{1}$. Assuming such an $a_{i}$ exists (that is, $a_{1} \neq 1$ ), begin cycle $C_{2}$ with $a_{i}$ and repeat the procedure, starting a new cycle every time we encounter a new smallest element. The resulting cycles (after inserting the distinguished one in its proper place) will be a permutation of $n$ elements written in our standard notation. Hence we have a one-to-one correspondence between the sets counted on both sides of Identity 4.

Notice that by distinguishing exactly $m$ of the cycles above, the procedure above can be easily modified to prove the more general

$$
\sum_{k=m}^{n}\left[\begin{array}{l}
n \\
k
\end{array}\right]\left(\begin{array}{l}
k \\
m
\end{array}\right)=\left[\begin{array}{l}
n+1 \\
m+1
\end{array}\right]
$$

Likewise by distinguishing an arbitrary number of cycles, the same kind of procedure results in

$$
\sum_{k=0}^{n}\left[\begin{array}{l}
n \\
k
\end{array}\right] 2^{k}=(n+1) !
$$

\section{Beyond harmonic numbers}

We have only scratched the surface of how combinatorics can offer new insights about harmonic numbers. Other combinatorial approaches to harmonic identities are presented by Preston [6]. We leave the reader with a challenge: A hyperharmonic number 
$H_{n}^{(k)}$ is defined as follows: Let $H_{n}^{(1)}=H_{n}$ and for $k>1$, define $H_{n}^{(k)}=\sum_{i=1}^{n} H_{i}^{(k-1)}$. Now consider the following generalization of identity (1) from The Book of Numbers by Conway and Guy [4]:

$$
H_{n}^{(k)}=\left(\begin{array}{c}
n+k-1 \\
k-1
\end{array}\right)\left(H_{n+k-1}-H_{k-1}\right) .
$$

Such an identity strongly suggests that there must be a combinatorial interpretation of hyperharmonic numbers as well. And indeed there is one [2]. You can count on it!

Acknowledgment. We thank Michael Raugh, David Gaebler, Robert Gaebler, and the referees for helpful comments, and Greg Levin for the illustrations. We are grateful to Janet Myhre and the Reed Institute for Decision Sciences for supporting this research.

\title{
REFERENCES
}

1. Robert Beals, personal correspondence, 1986.

2. Arthur T. Benjamin, David J. Gaebler, and Robert P. Gaebler, A Combinatorial Approach to Hyperharmonic Numbers, preprint.

3. Louis Comtet, Advanced Combinatorics, D. Reidel Publishing Company, Boston, 1974.

4. John H. Conway and Richard K. Guy, The Book of Numbers, Copernicus, 1996.

5. Ronald L. Graham, Donald E. Knuth, and Oren Patashnik, Concrete Mathematics, Addison Wesley, 1993.

6. Greg Preston, A Combinatorial Approach to Harmonic Numbers, Senior Thesis, Harvey Mudd College, Claremont, CA 2001.

\section{Giraffes on the Internet}

\author{
RICHARD SAMUELSON
}

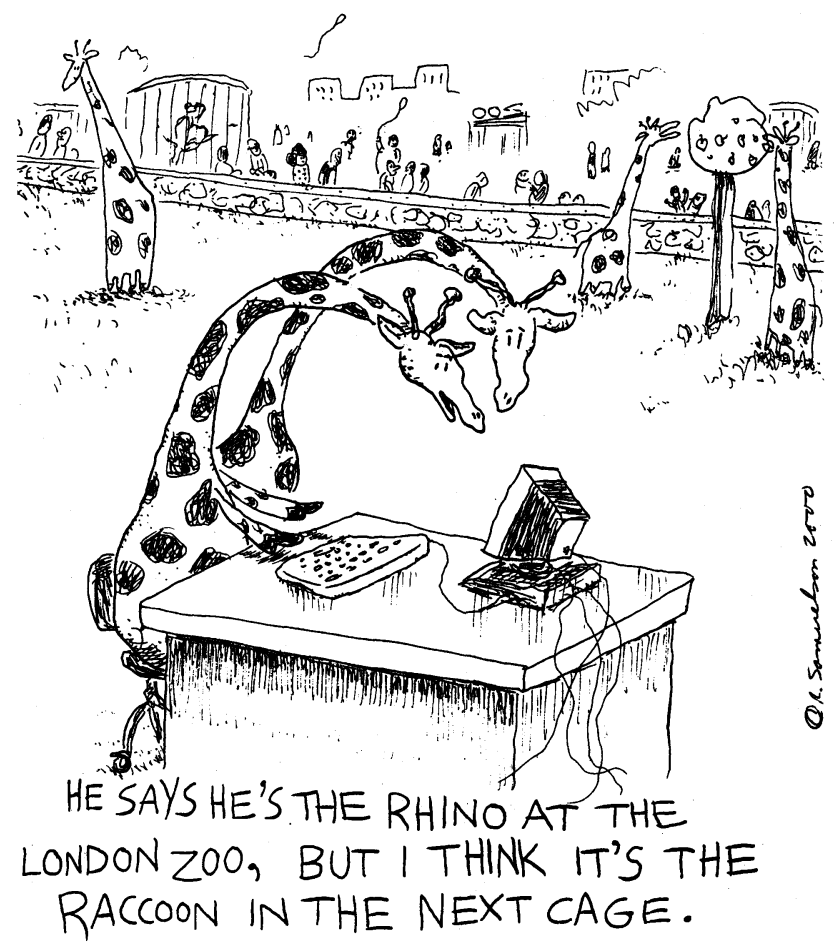

